\title{
Refsum Disease
}

National Institute of Neurological Disorders and Stroke (NINDS)

\section{Source}

National Institute of Neurological Disorders and Stroke (NINDS). Refsum Disease

Information Page.

Adult Refsum disease (ARD) is a rare genetic disease that causes weakness or numbness of the hands and feet (peripheral neuropathy). Due to a genetic abnormality, people with ARD disease lack the enzyme in peroxisomes that break down phytanic acid, a type of fat found in certain foods. As a result, toxic levels of phytanic acid build up in the brain, blood, and other tissues. The disease usually begins in late childhood or early adulthood with increasing night blindness due to degeneration of the retina (retinitis pigmentosa). If the disease progresses, other symptoms may include deafness, loss of the sense of smell (anosmia), problems with balance and coordination (ataxia), dry and scaly skin (ichthyosis), and heartbeat abnormalities (cardiac arrhythmias). Some individuals will have shortened bones in their fingers or toes, or a visibly shortened fourth toe. Although the disease usually appears in early childhood, some people will not develop symptoms until their 40 s or 50 s. 